Original Research

\title{
'A Way of Being in the World': An Exploration of the Experience of Developing Self-Compassion through Online Training
}

Jack Deacon ${ }^{1}$, Clare Northover ${ }^{1}$, John King ${ }^{2}$, Chris Irons ${ }^{3, *}$

1. Trainee clinical psychologist at University College London, London, UK; E-Mails: j.deacon.17@ucl.ac.uk; clare.northover.18@ucl.ac.uk

2. Research director at University College London, London, UK; E-Mail: john.king@ucl.ac.uk

3. Director and clinical psychologist at Balanced Minds, London, UK; E-Mail: chris@balancedminds.com

* Correspondence: Chris Irons; E-Mail: chris@balancedminds.com

Academic Editor: Gerhard Litscher

Special Issue: Compassion Focused Therapy (CFT) - New Insights and Outcomes

OBM Integrative and Complementary Medicine

2021, volume 6 , issue 4

doi:10.21926/obm.icm.2104050
Received: September 21, 2021

Accepted: November 12, 2021

Published: November 23, 2021

\begin{abstract}
Compassion Focused Therapy (CFT) has increasingly been shown to have a positive impact for people experiencing a range of difficulties, and online self-help formats could provide a way of increasing its accessibility and scalability. This study explored adult participants' subjective experiences of engaging with online self-compassion training drawn from Compassionate Mind Training (CMT) and CFT. Semi-structured interviews were conducted with 15 participants who had completed online self-compassion training, and the interview data was analysed using reflexive thematic analysis. The analysis generated four main themes and 17 sub-themes. The four main themes generated were ' $A$ way of being in the world', 'Old habits die hard', The learning process, and Context and resources. These themes showed that while the process of developing self-compassion was often challenging, most participants described experiencing positive changes in their ways of relating to themselves and others. Participants identified many facilitators and barriers to change, including factors related to the training
\end{abstract}


format and their wider social contexts. These findings support previous research into CMT and CFT, particularly in relation to some common positive changes people experience and some of the difficulties with this process (e.g. fears of compassion). Participants' descriptions offer promise that approaches based on CMT and the CFT model can be experienced as acceptable and helpful by people engaging through an online self-help format as well as through individual and group-based interventions. The facilitators and barriers to change described by participants indicate the strong influence of people's contexts and the need for these to be carefully considered when designing and implementing approaches.

\section{Keywords}

Compassion; compassionate mind training; compassion-focused therapy; online interventions; qualitative research

\section{Introduction}

This study explored adult participants' subjective experiences of engaging with online selfcompassion training based on Compassionate Mind Training (CMT) [1], which is drawn from the Compassion Focused Therapy (CFT) model [2].

Researchers are increasingly exploring people's subjective experiences of engaging with compassion and CFT-based interventions. In Pauley and McPherson's exploratory study about participants' experiences of and perspectives towards compassion and self-compassion, compassion was described as an active and kind process which was considered meaningful and potentially helpful for people's difficulties, but also one that it is difficult to engage in [3].

These findings came after previous research had similarly highlighted fears, doubt and resistance towards compassion in a group of people experiencing chronic mental health difficulties $[1,4]$. Fears in Gilbert and Procter's study were related to "whether compassion was deserved, or a weakness, unfamiliarity with compassion, unresolved grief of wanting love and kindness but often feeling lonely and rejected, and simply 'never considering the value of self-compassion'" (p. 243) [1].

Such aversive reactions to compassion inevitably have significant implications for therapeutic practice and they are often explicitly explored in CFT. Indeed, Gilbert, McEwan, Matos and Rivis developed a scale - now often used in CFT research and practice - specifically for measuring fears of compassion from others, for others, and for oneself [4]. A meta-analysis based on fears of compassion showed significant associations between fears of compassion and mental health difficulties [5].

While it is often difficult for people to engage with compassion and self-compassion, in many studies participants have described being able to overcome barriers they faced [6-8]. Having a supportive relationship with therapists or group facilitators has often been described as essential to this process of overcoming barriers and developing self-compassion $[7,9,10]$.

Studies have also demonstrated positive aspects of participants' experience with CFT, such as becoming more able to step back from difficulties, taking a non-blaming attitude to oneself, gaining insight into difficulties, engaging in compassionate behaviours and seeing their difficulties as part of a shared human experience $[8,11,12]$. Gilbert has written about compassion being a social 
mentality [13] and this has been supported by participants reporting an increased sense of 'common humanity' that Neff emphasises in her conceptualisation of self-compassion [14]. The integrity of this social dimension to CFT is further supported by the number of participants who have described CFT helping them develop a new way of relating to others as well as themselves $[11,15]$.

\subsection{Compassionate Mind Training}

CFT incorporates Compassionate Mind Training (CMT), which includes some of the skills and strategies used in individual and group-based CFT. CMT can sit outside of therapy without the common aspects of therapeutic relationships such as boundary-setting, transference, countertransference, formulation-making and goal-setting [16].

CMT starts with psychoeducation focused on the definition of compassion, common blocks and fears, and the idea of compassion having three 'flows' (self-compassion, self to other and other to self). It then explores the evolutionary roots of many difficulties people experience, with an emphasis on a non-blaming understanding of these leading us to have 'tricky brains'. People learn about the three emotional systems and how the cultivation of the soothing system can help to regulate distress [13]. People are then encouraged to try a range of practices that are ultimately aimed at cultivating a compassionate self-identity. This 'compassionate self' is associated with qualities such as strength, wisdom and caring motivation, and it is used to manage daily struggles [16].

\subsection{Self-Help Applications}

While there is a significant body of research supporting CFT in one-to-one and group formats, there is less research looking into its application in self-help formats which could increase the scalability and accessibility of CFT $[17,18]$. We are in an era in which self-help formats are receiving increased attention from healthcare provides, and other approaches such as CBT are routinely offered via online guided self-help programmes in the NHS. Reviews and meta-analyses have shown that self-help interventions can have positive effects on various mental health difficulties such as depression and anxiety $[19,20]$. Findings suggest that guided self-help interventions (i.e. involving some support from a practitioner) may be more effective than unguided self-help interventions [18, 20].

The small number of trials researching CFT-based self-help interventions have had some encouraging results showing positive effects on emotional well-being, self-compassion and depressive symptoms [21-26]. These studies have mostly had specific target populations (e.g. people who have received a diagnosis of an eating disorder) and have had various methodological limitations, such as small sample sizes and a lack of follow-up. Halamová, Kanovský, Pačutová and Kupeli's study was the first to test the effectiveness of CMT delivered as an online self-help intervention for a non-clinical sample [21]. The findings showed significant reductions in negative thoughts and feelings related to self-criticism for the CMT group, which were still present at a twomonth follow-up. 


\subsection{Study Aims}

CMT self-help interventions are at an early stage of their development, despite some encouraging findings in the handful of studies that have piloted such an approach. To our knowledge, there have been no qualitative studies on this topic yet so there is an important gap in the literature that this study targeted.

This study was focused on participants' subjective experiences of engaging with online selfcompassion training developed for the general population by $\mathrm{Cl}$. This approach is currently at a piloting stage so an inductive approach was chosen, based on semi-structured interviews and a reflexive thematic analysis of the interview data.

Research question: What are participants' subjective experiences of engaging with the selfcompassion training?

\section{Materials and Methods}

\subsection{Participants and Procedure}

This research study was conducted with Northover, Deacon, Irons and King and their article in this edition has already provided information that will not be repeated here. While some parts of our study were conducted together (e.g. study conception and recruitment), we had different emphases in our respective data collection and analysis processes. CN collected and analysed quantitative data in Northover et al.'s study, while in this study JD conducted semi-structured interviews and a reflective thematic analysis of the qualitative interview data. As such, this paper will be focused on the JD's data collection and analysis.

As the self-compassion training was being piloted and at this stage does not have a particular target demographic, we chose to recruit adults from the general population, using convenience sampling and snowballing techniques. JD and $\mathrm{CN}$ advertised the study through social media (Facebook and Twitter) on various community groups, with the aim of reaching a diverse range of people. In order to try to maximise the number of participants that could take part, we chose broad inclusion criteria: participants had to be over 18 years old, have regular access to the internet and be able to read English fluently.

When signing up for the study, participants were asked to specify whether they would be willing to take part in a follow-up interview about their experience of the training. An interview information sheet and consent form were emailed to all participants who had consented to be contacted. The only addition to the inclusion criteria for the interviews was that participants needed to have completed the training by the time they engaged in an interview. Out of the 72 participants invited to take part of an interview, 15 consented and all of these were interviewed. Participants demographic details are provided in Table 1.

Table 1 Participant demographics.

\begin{tabular}{lllll}
\hline Participant & Age & Gender & Ethnicity & Highest level of education \\
\hline 1 & 41 & W & White British & Doctorate \\
2 & 27 & W & White Latvian & Master's \\
3 & 39 & W & White British & Master's \\
\hline
\end{tabular}




\begin{tabular}{lllll}
\hline 4 & 41 & W & White British & PhD \\
5 & 29 & W & White Irish & Doctorate \\
6 & 28 & W & White Greek & Degree \\
7 & NS & W & NS & NS \\
8 & 42 & M & White British & Degree \\
9 & NS & M & White British & NS \\
10 & 33 & W & White British & Degree \\
11 & NS & W & NS & Doctorate \\
12 & 51 & W & White British & Master's \\
13 & 30 & W & White British & Degree \\
14 & 49 & W & White British & Post-graduate diploma \\
15 & NS & W & NS & PhD \\
\hline
\end{tabular}

Key: $W=$ woman, $M=$ man and NS=not specified.

Braun and Clarke suggest 6-15 as a 'rule of thumb' for a medium-sized thematic analysis project (including doctoral theses) [27] and we decided to reach the higher limit of this suggestion in an attempt to generate a rich amount of data. Due to the limited time available for data collection and analysis, it was not possible to further extend data collection beyond this.

\subsection{Self-Compassion Training Content}

The self-compassion training content was developed by $\mathrm{Cl}$ and based on $\mathrm{CMT}$, which is drawn from CFT theory, practice and outcome research [24, 28, 29]. The training lasted four weeks, each with a different emphasis. On a day of the week agreed with each participant, they were emailed a prompt and a Qualtrics link to access:

- A video (approximately 30 minutes) in which $\mathrm{Cl}$ explained CFT-based ideas and exercises.

- A 1-2 page handout summarising the content of the videos.

- An audio exercise to practise throughout the week.

Below is a summary of what each week covered:

Week one: This session provided some definitions of self-compassion, explored why selfcompassion is important and described how to lay foundations for self-compassion by understanding Gilbert's affect regulation model in CFT (i.e. three systems: the threat, drive and sooth systems) [30]. $\mathrm{Cl}$ then encouraged participants to engage in an exercise called soothing rhythm breathing, which is aimed at accessing and developing the parasympathetic nervous system. This has been found to be associated with threat regulation and social connection [30]. Participants were asked to practice soothing rhythm breathing every day over the next week. They were also asked to try to notice which of the three systems they found themselves in, and to ground themselves using the soothing rhythm breathing.

Week two: This session explored the concept of building a compassionate self. It focused on qualities this compassionate self might have: caring-commitment, wisdom, strength and courage. $\mathrm{Cl}$ talked about how to begin directing this sense of compassion and goodwill to oneself. This practice has been found to be linked with increases in self-compassion, and reductions in shame and stress $[24,31]$. Participants were asked to practice with an audio exercise focused on holding a caring intention towards oneself, as well as linking this back to the affect regulation model introduced in the previous week. 
Week three: This session involved $\mathrm{Cl}$ introducing compassion as a relational concept and guiding participants to try to deepen their compassionate relationship with themselves through 'compassionate other' imagery [28, 29]. Participants were asked to think about the qualities that a 'compassionate other' would embody and to hold this image in mind, particularly in terms of how their presence would feel. $\mathrm{Cl}$ encouraged participants to practice this as well as moments of 'CARE' ( $C$ = connect with your compassionate self; $A=$ allow yourself to slow down for a moment; $R$ = reflect on how you are in this moment; and $E$ = extend a sense of care and kindness to yourself).

Week four: The final session was focused on helping participants to think about how they could apply self-compassion in their everyday lives. $\mathrm{Cl}$ emphasised the need for practice and talked about steps people could take in in trying to switch from their 'threat mind' to their 'compassionate mind'. He also described how self-compassion can be embodied in compassionate letter-writing, which utilises adaptations to expressive writing to help people build a more compassionate relationship with themselves [32]. Participants were encouraged to practice both strategies, as well as continuing to draw upon ideas and strategies covered in the previous weeks.

\subsection{Data Collection}

Interviews were conducted by JD on Microsoft Teams. A semi-structured interview schedule was developed based on our research questions and in line with relevant methodological guidelines [27]. A semi-structured approach was chosen in order to strike a balance between participant-driven and researcher-driven content. Though we spoke about some broad areas it would be helpful for the interviews to cover in trying to meet the research aims, we did not want to rigidly impose these on the interview space. The interview schedule was therefore used as a loose aide memoire regarding some areas relevant to the research aims. JD mostly used open questions and tried to create a relatively informal, comfortable atmosphere in which participants felt empowered to speak openly [33].

\subsection{Data Analysis}

\subsubsection{Reflexive Thematic Analysis}

We chose to analyse the data using reflexive thematic analysis [34]. Reflexive thematic analysis offers a flexible approach for providing rich and detailed accounts of data, and it can be utilised from various epistemological positions. It can be used effectively to examine similarities and differences between research participants' perspectives, to summarise key features of large data-sets, and to inductively generate themes from the data $[35,36]$. For these reasons, we decided that it was wellsuited to our aim of inductively exploring participants' subjective experiences of the selfcompassion training, and writing up the findings in a way that would identify shared patterns of meaning as well as differences within the data.

The analysis went through the following phases, in a recursive process which involved moving back and forth between them [37]:

Familiarisation with the data: JD read and re-read the data in order to become immersed and intimately familiar with its content. 
Coding: JD generated codes that identified features of the data that seemed relevant to answering the research question. JD coded the entire dataset and then collated all the codes and relevant data extracts for the next stages of analysis.

Generating initial themes: JD examined the codes and collated data to develop broader patterns of meaning. JD then collated data related to the possible themes, in order to review their viability. A fellow Trainee Clinical Psychologist at UCL (MVW; see acknowledgements) independently coded two of the interviews and the similarities and differences between our coding were reviewed in relation to the possible themes.

Reviewing themes: JD checked the possible themes against the dataset, to gauge whether they told a convincing story of the data and answered the research question. In this phase, themes were refined, which involved them being combined, split or discarded. Themes were seen as a "pattern of shared meaning underpinned by a central concept or idea" in line with Braun and Clarke's definition (p. 845) [37]. The reviewing and refining of themes were discussed by JD and JK.

Defining and naming themes: JD developed a detailed analysis of themes, working out the scope and focus, and determining their 'story'. Informative names were generated for the themes.

Writing up: In this final phase, JD weaved together the narrative with data extracts, and contextualised the findings of the analysis in relation to existing literature.

\subsubsection{Reflexivity}

Qualitative research tends to emphasise researchers considering the role of their involvement in influencing and informing studies [28]. By engaging with these forms of reflexivity, it is possible to move towards an increased awareness of the researcher's role in co-constructing knowledge [38]. An overview of the lead author's engagement with reflexivity is provided below for the sake of concision, and because he conducted most of the data collection and analysis. He carried out bracketing interviews and kept a reflexive journal throughout the research process in order to help hold in mind pre-conceptions and strive to conduct the research inductively, while recognising that this did not make him a 'blank slate' [39].

Epistemological reflexivity. JD adopted a critical realist position in this study, reflecting his assumption that the world is objective and real, but it is not possible to make direct contact with this reality and our perceptions of it are shaped by factors including beliefs, language, previous experiences and social contexts [40-42]. This influenced the study in a number of ways. For example, JD conducted bracketing interviews and reflexive journals in order to take a sceptical and contextualised perspective towards the way he was conducting interviews and analysing the data, rather than trying to altogether 'remove' the influence of his subjectivity. Furthermore, the concept of 'saturation' was not used when deciding sample size. From a critical realist position that assumes the partial and co-constructed nature of knowledge, it is arguably not possible to reach a point of theoretical 'knowingness' where it can be confidently assumed that no further information will be generated [37].

Personal reflexivity. JD was in part drawn to conducting this research project because of positive experiences of drawing upon CFT in his clinical work, as well as resonating personally with a lot of the difficulties at which CFT is targeted. As such, he was aware of already having a positive attitude towards CFT-based approaches and optimism about the potential benefits of participants engaging 
with the training. However, he was also sceptical of how helpful clients would find CMT without any tailoring to individuals. This scepticism was partly informed by clinical experience of clients sometimes finding CFT-based strategies difficult, and also concern about the trajectory of mental health services offering cost-effective approaches at the expense of other factors that are known to often be crucial, such as the therapeutic relationship. Ethical approval was obtained from UCL Ethics Committee (ref: CEHP/2020/581) on 1/08/2020.

\section{Results}

The reflexive TA generated four main themes and 17 sub-themes. The four main themes generated were 'A way of being in the world', 'Old habits die hard', The learning process, and Context and resources. The themes are visually represented in Figure 1, and the distribution of themes among the 15 participants is represented in Table 2.

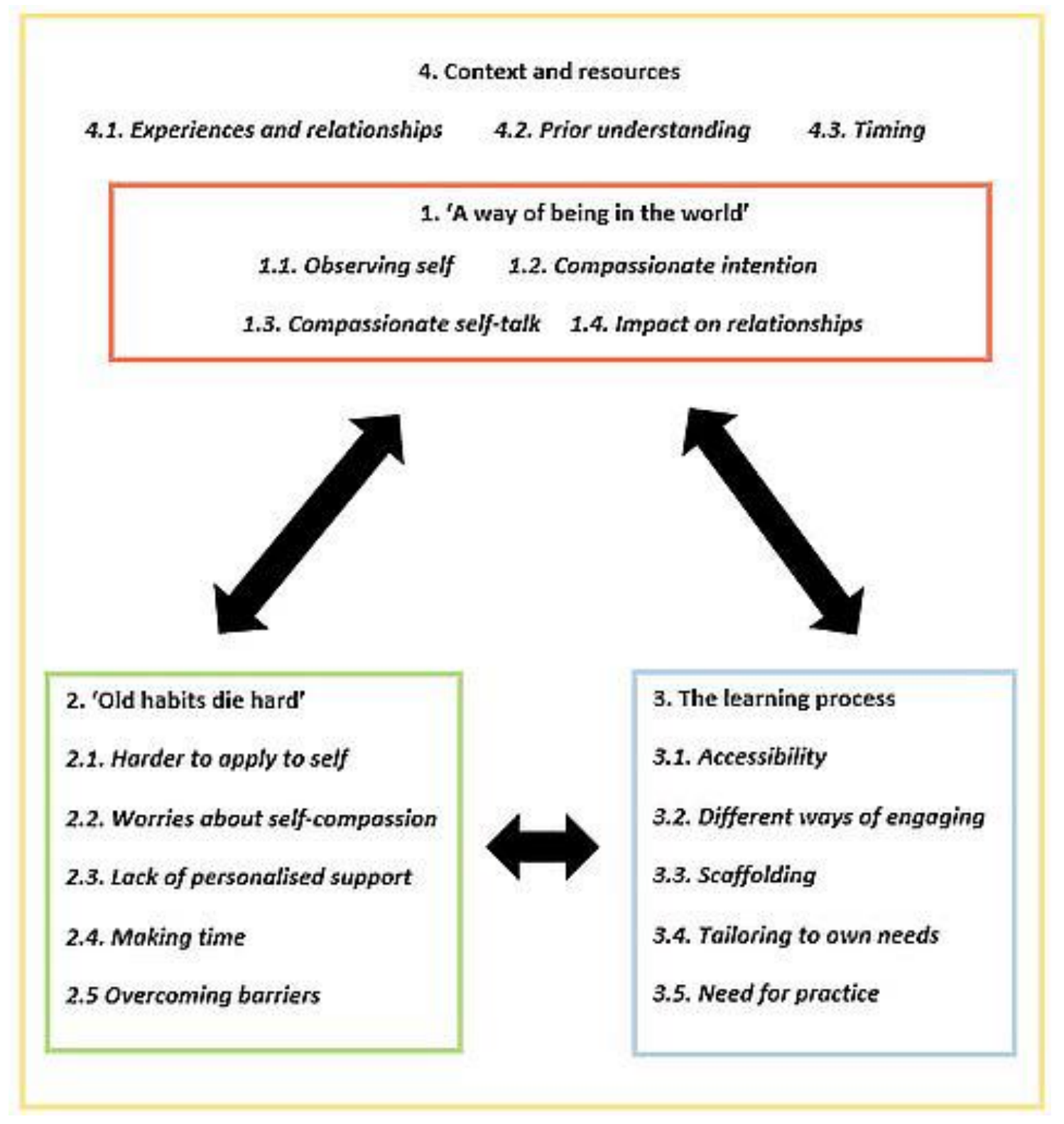

Figure 1 Visual representation of themes. 
Table 2 Distribution of themes among participants.

\begin{tabular}{|c|c|c|c|c|c|c|c|c|c|c|c|c|c|c|c|}
\hline Main themes & Sub-themes & 12 & 3 & 4 & $\mathbf{5}$ & 6 & 7 & 8 & 9 & 10 & 11 & 12 & 13 & 24 & 15 \\
\hline \multicolumn{16}{|c|}{ 1. 'A way of being in the world' } \\
\hline & 1.1. Observing self & & & & & & & & & & & & & & \\
\hline & 1.2. Compassionate intention & & & & & & & & & & & & & & \\
\hline & 1.3. Compassionate self-talk & & & & & & & & & & & & & & \\
\hline & 1.4. Impact on relationships & & & & & & & & & & & & & & \\
\hline \multicolumn{16}{|c|}{ 2. 'Old habits die hard' } \\
\hline & 2.1. Harder to apply to self & & & & & & & & & & & & & & \\
\hline & 2.2. Worries about self-compassion & & & & & & & & & & & & & & \\
\hline & 2.3. Lack of personalised support & & & & & & & & & & & & & & \\
\hline & 2.4. Making time & & & & & & & & & & & & & & \\
\hline & 2.5. Overcoming barriers & & & & & & & & & & & & & & \\
\hline \multicolumn{16}{|c|}{ 3. The learning process } \\
\hline & 3.1. Accessibility & & & & & & & & & & & & & & \\
\hline & 3.2. Different ways of engaging & & & & & & & & & & & & & & \\
\hline & 3.3. Scaffolding & & & & & & & & & & & & & & \\
\hline & 3.4. Tailoring to own needs & & & & & & & & & & & & & & \\
\hline & 3.5. Need for practice & & & & & & & & & & & & & & \\
\hline \multicolumn{16}{|c|}{ 4. Context and resources } \\
\hline & 4.1. Experiences and relationships & & & & & & & & & & & & & & \\
\hline & 4.2. Prior understanding & & & & & & & & & & & & & & \\
\hline & 4.3. Timing & & & & & & & & & & & & & & \\
\hline
\end{tabular}

Key: Filled squares indicate that there was direct evidence for the corresponding theme in an interview with a participant. Participant numbers correspond to those in Table 1.

In order to give emphasis to participants' own descriptions of their experiences with the training, we have presented a verbatim excerpt from interviews corresponding to each theme. We have chosen excerpts based on how well we believe they illustrate their corresponding themes. Ellipses (...) are used when parts of interviews are omitted, and we have inserted text within square brackets [ ] to provide some clarifications. We have used participant numbers in order to preserve confidentiality.

\section{4. 'A Way of Being in the World'}

This theme speaks to the way in which the training not only provided tools that can be applied reactively, but a mentality or worldview that one can bring to oneself and others across different areas of life. The sub-themes below specify some of the aspects of this mentality that many of the participants described. Table 2 shows that this theme and its sub-themes were not supported by the interview with Participant 12, though she did have a positive experience of the training. She talked instead of the training giving her some tangible ways of putting ideas she was already familiar with into practice, and how the compassionate friend imagery reduced her sense of aloneness with her difficulties. 
"I suppose the thinking has got to be that it is not a reactive cure to a bad thing. It should be sort of a way of being in the world." (Participant 13)

\subsection{Observing Self}

Participants talked about the training helping them to become more able to take a step back and observe their difficulties without feeling so consumed by them. This helped them gain insight into some of their automatic responses to difficult situations and to feel more able to think about how they might break out of these patterns in the moment. This was often challenging, and they emphasised the fact that this awareness of their thought processes or emotional experience usually lagged behind their automatic responses. Participants were sometimes able to 'catch' themselves in the moment, but sometimes it was only afterwards that they were able to reflect on their internal and external responses. Nonetheless, the self-compassionate mentality seemed to offer participants a sense of warmth and openness that helped them tune into their experience.

"Sometimes I noticed that I wasn't being my compassionate self. And I was like, 'Oh, that wasn't very compassionate of me'. That critical self was the opposite of what I was meant to do. But I suppose that exercise [compassionate self exercise] was really helpful. It helped me stop and think, like, 'Am I being my compassionate self in this situation?'”' (Participant 2)

\subsection{Compassionate Intention}

The compassionate intention participants described bringing to their lives was meant that when they observed that they had become caught in an unwanted way of relating, this would often be followed by asking themselves 'How can I respond more compassionately in this situation?'. This intention seemed to be an important part of developing a new 'way of being in the world' as it speaks to a certain stance that participants were striving to take. Similarly to the previous subtheme, though, participants described this intention as being something that is not a constant. Instead, it is something that easily slips out of awareness and there is a continuous oscillation between other things preoccupying one's mind and then re-discovering this intention.

"When I noticed I'm in that unhelpful place of being self-critical with how I'm thinking, is thinking, 'OK, what would the wise, strong and caring me say in this situation, or how can I engage that way of thinking instead?' Even if I don't believe it, at the same time trying to access it more." (Participant 15)

\subsection{Compassionate Self-Talk}

Compassionate self-talk was one of the main ways in which participants described putting into practice some of the ideas and strategies that the training introduced. This often took the form of noticing self-critical thoughts and generating a more compassionate alternative to these. Participants also talked about finding it helpful to imagine what they might say to a friend who was in their shoes, and to try to turn this more compassionate response towards themselves. The compassionate self-talk frequently seemed to comprise a non-blaming acknowledgement of what they were experiencing, and a validating re-frame of a self-critical thoughts (e.g. 'It's understandable that you feel like this' or 'You've tried your best'). 
"I think even today, I found myself thinking 'It's understandable that it would be difficult because the IT has gone down, it's the middle of the pandemic and I've got loads to do'. So it's giving me permission. A little bit of 'Well, just see what we can do. And that's all we can do really.'" (Participant 14)

\subsection{Impact on Relationships}

Participants described the training having an impact on their relationships with others as well as themselves. Participants described carrying their compassionate intention into their interactions and noticing that this prevented misunderstandings and disagreements from turning into arguments. They also talked about sense of prioritising the relationship over proving a point. One participant talked about how they had been a 'people-pleaser' throughout their life and they were gradually becoming more able to value their own needs as much as others. Participants who had used CFT in their work with clients said that the training had enhance their appreciation of how difficult it can be to internalise and integrate these ideas, which they felt would lead to them having more understanding and flexibility in their clinical work.

"So being able to be more kind to myself, I think that would be the basis of it. Being able not to fall into that kind of cycle of negative thinking about myself about being to blame. It also helped us to get out of the interpersonal dilemma. The fact that I didn't spiral it in my mind meant it didn't spiral so much between us." (Participant 15)

\section{5. 'Old Habits Die Hard'}

While most participants described noticing tangible benefits from engaging in the training, they also all described experiencing challenges in their attempts to become more self-compassionate. This theme is titled 'Old habits die hard' because it a phrase that a couple of participants said, and a phrase which captures the sentiment of what was described by many others. Perhaps unsurprisingly, most of the participants interviewed were drawn to the training because they identified self-compassion as something that they were lacking, and it is clearly often hard to develop when it seems so far removed from people's typical ways of relating to themselves.

"I guess old habits die hard. So there's something there thinking maybe I can motivate myself by being more critical, by using anxiety just because that's been a pattern that I've probably used a lot of my life." (Participant 10)

\subsection{Harder to Apply to Self}

One of the most frequently-described challenges that participants described was that compassion is harder to apply to oneself than it is to apply to others. It seemed that it was often hard for participants to prioritise their emotional wellbeing enough to direct a compassionate intention towards themselves and, when they did manage to do this, some worried that it would have negative consequences on their relationships and performance at work (e.g. people would stop liking them and/or they would stop making an effort). Participants described there being a considerable gap between understanding these ideas on an intellectual level and being able to engage with them on a more emotional level when applying them to yourself. 
"I guess this is the whole thing about self-compassion - when I speak to friends who are going through a difficult time, I'm really understanding, I'll really talk them through it, I'm really kind. But the moment I've done something that I don't think is good, or I've made a bad decision or something, I'm instantly very critical." (Participant 9)

\subsection{Worries about Self-Compassion}

Participants described worries about self-compassion making it hard for them to try to engage with the training. Some worried that it would resemble a form of self-indulgence and they would end up disengaging from responsibilities. One participant described worrying that if they were to become more self-compassionate, this might jeopardise their relationships as they were so used to taking a passive role and prioritising the needs of others at their expense of their own. A different participant saw self-criticism as a way of protecting others from the anger they experienced in difficult situations and they worried that if they were to become more self-compassionate, the anger they usually directed inwards would be turned outwards and their relationships would not be able to survive this.

"I do sort of worry that if I have enough soothing, then the drive might disappear and it might be that I don't really register threats anymore. But also, I won't have the get up and go either." (Participant 11)

\subsection{Lack of Personalised Support}

Another thing that made it hard for some participants to develop self-compassion was the lack of personalised support. The self-help format of the training meant that there was no opportunity for individual meaning-making, support from a professional nor peer support. The support that participants said they would have liked included a check-in before starting to help think about why self-compassion might be important for them personally, a check-in between sessions to help overcome any barriers and to remind/prompt them to practice exercises, and a follow-up conversation to help them think about how they would take the training forward. Several participants reflected that it was hard to maintain the motivation to continue practicing the ideas and exercises, and that having somebody check in with them would have brought more accountability to engage with the training.

"I suppose at the end of the day, nothing really beats having somebody doing it on a personal level with you, who's listening to your story and tailoring it to you, because I guess with the online training, there's no background to it. Like, you know, I can acknowledge that self-compassion is important, but there's no 'Why is the self-compassion important for you? What other things might need to be put in place to make sure that it's most effective?'” (Participant 3)

\subsection{Making Time}

Difficulties with making time to practice self-compassion were talked about by most participants, many of whom described it as the thing they found most difficult about the training. While some participants said that their sense of lacking self-compassion acted as a motivator to regularly practice, others described it getting in the way as it inherently made it harder for them to prioritise their wellbeing enough to engage with the training. A lot of the participants led busy lives and when 
they were able to make time to engage with the training, this often necessitated having flexibility in how they fitted it into their schedules and how they prioritised exercises they found most helpful. As mentioned in the previous sub-theme, the lack of accountability made it particularly hard for some participants to generate motivation.

"Because this is a thing you do for you, it goes go to the bottom of the pile, because there's nobody demanding it. And if your self-compassion is low you're definitely not going to prioritise it." (Participant 3)

\subsection{Overcoming Barriers}

Several participants talked about finding ways to overcome some of the barriers they experienced. For the participants who overcame worries about self-compassion, they talked about the training helping them to re-frame self-compassion as something that is not simply 'letting oneself off the hook', but rather something that can motivate them towards their goals and values in a more warm and supportive way than their typically self-critical and perfectionistic ways of relating to themselves. Some participants who struggled to make time for the training talked about creatively finding ways of establishing their own practice, such as using the training's exercises as and when they felt the need for them. A couple of participants who had reflected on the lack of personalised support also reflected that this helped them to establish more of an intrinsic motivation for doing the training, which Participant 10 referred to as "Doing it for me".

"And for me and I guess the process of change, I would expect requires some degree of working through some negative emotions. If it was just positive, I'm not sure I would have been doing what I needed to do to get benefits from it . . And implicitly, somewhere in my goals for it, I was hoping that it would bring up difficult feelings so that I truly am making a change in what's causing me difficulty in day-to-day life. But no more difficult feelings than other situations in my life. So it's not like it was causing distress that I wouldn't have otherwise had in certain moments. And it was giving me ways to overcome some of the things causing me those upsetting feelings." (Participant 15)

\section{The Learning Process}

The third main theme generated was the learning process of engaging with the training and trying to develop self-compassion. Participants described their experience of certain factors which seemed to influence the extent to which they could take in, and take forward, the training's ideas. Some of these factors will be elaborated upon below, including the training's accessibility, use of different types of materials, and the need for participants to find ways of tailoring the training to their needs. As will be outlined in the final theme, participants came to the training from different positions in their lives (e.g. different levels of prior understanding of self-compassion and different levels of motivation for developing it). This theme and its sub-themes touch upon some of the things that seemed to have interacted with these different positions and people's engagement with the training.

"I used to be quite a perfectionist, which I'm not anymore, I don't really think I am much. Initially going through that process was like, well, 'I have to do it really well and I have to get it right. And there must be a solution. And it was a must be one solution. That's my solution that works for me'. When actually... It doesn't matter if it doesn't work first time, but try different things. " (Participant 3) 


\subsection{Accessibility}

For the most part, participants found the training highly accessible and few reported difficulties understanding the training's materials. Participants said that the meditation exercises were easy to follow and that the four weeks of 30-minutes sessions was a reasonably manageable commitment. However, some participants also talked about there being some ways in which the study's accessibility could be improved. A couple of participants suggested that some people might require greater explanation of certain concepts and more time to digest concepts before moving on to the next ones. There were also some issues with how the study was set up online, such as not being able to easily access previously-covered materials. This latter issue relates to how the study had to be set up for research purposes of data collection and participant anonymity.

"I liked the fact that it was short. Like I wouldn't have liked it to be longer, maybe because it would be even more time to find. And the fact that they gave us to read what was in the video, I found very helpful because I can go back and read them." (Participant 6)

\subsection{Different Ways of Engaging}

The training's use of various formats (30-minute videos, written handouts and brief audio exercises) was something that seven participants said they valued about the training. This was seen to help them digest the ideas and tailor their engagement with the training to their own needs. For example, the written handouts provided a way of reminding themselves of the ideas without having to re-watch the videos, and the brief audio exercises provided an accessible way of practising tuning into a compassionate stance. Many participants also found that they did not click with one of the formats, so the use of multiple formats meant that they could choose how to make use of the materials they found most helpful.

"But the pictures and the reading was, like, really simple, straightforward. So, for example, though the video sometimes felt long, then I went on to the reading and stuff and I thought, 'Oh, that's everything he said'. And that was really straightforward and quick, but the video is really helpful just because hearing him say it in a really calm voice is really helpful." (Participant 2)

\subsection{Scaffolding}

Another key part of the learning process for nine of the participants was the way in which the learning was scaffolded. Participants varied in how familiar they were with the training's ideas, so it was helpful to have the material gradually introduced and built upon throughout the four weeks. The fact that the various materials were relatively short was helpful too, as this allowed people to easily to return to what was covered in previous weeks and to not feel overloaded by the amount of information they were taking in. Participants also talked about feeling reassured by $\mathrm{Cl}^{\prime} \mathrm{s}$ comments about certain exercises (e.g. that it is normal for the mind to wander during audio exercises), as these made them less likely to take a self-critical stance towards how well they were doing the exercises.

"I liked the way it started off with just the soothing rhythm breathing. And then it introduced other exercises along the way and they all kind of coupled together, merged together. And so it was a helpful, gradual way of learning about the different techniques and how to incorporate them together." (Participant 2) 


\subsection{Tailoring to Own Needs}

Most participants described the learning process involving tailoring the training to their own needs in various ways, some of which have been mentioned in previous themes. For example, it was not always realistic to complete the training on the same day(s) each week so they had flexibility with themselves in terms of when they could fit it into their schedules. One participant said that the 10-minute audio exercises felt too long for her so she instead found a shorter version online. Some exercises elicited markedly different responses across participants, who sometimes described not 'clicking' with them. Rather than disengaging with the training altogether, though, they tended to decide to develop their own practice built from the exercises they found most beneficial.

"Like sometimes if I was feeling really exhausted, it was really good to be in meditation and choosing when I was going to do it myself... So I actually learnt that that flexibility was actually probably what I needed rather than rigidly saying do this every day, in spite of my initial concerns." (Participant 1)

\subsection{Need for Practice}

This sub-theme is interconnected with many of the themes already outlined, such as the 'Old habits die hard' and its sub-themes which showed how challenging it can be for people to develop self-compassion. The process therefore necessitates a lot of practice and for the intellectual understanding of concepts to be embodied in the choices people make in the ways they are relating to themselves and others. While some participants were pleased to report feeling that selfcompassion was gradually becoming more internalised and automatic, they also expressed worries that they would struggle to maintain the changes they had managed to make. Self-compassion was not seen as a way of being that is achieved once and for all, but as something that fluctuates and has to be striven towards on an ongoing basis.

"I appreciate how much regularity and persistence and the sort of the repetition of these things has an effect. We are our habits, literally, what you subscribe to, what you surround yourself by... it reinforces who I am and what I am. And so if I am bad habits, then that will reflect in my life. So for me, I appreciate the need to... To be well, I need to practise and it's not just sort of, you know, 'Go get a tablet'. It's something you've got to work on. And it's like 24/7, 365." (Participant 13)

\section{Context and Resources}

The final theme generated by the analysis pertains to the role of participants' contexts and resources in their engagement with the training. As touched upon previously and elaborated upon in the subsequent sub-themes, participants' experiences of the training were shaped to some extent by factors such as family backgrounds, cultural beliefs, work environments, prior familiarity with concepts, previous therapy and their emotional states. These factors had varied effects and levels of impact, and though it is inevitably hard to determine these with any precision, it was clear from the interviews that they played an important role for many participants.

"It's almost a societal thing rather than just an individual thing, I think. We're all -for whatever reason - programmed to be quite critical, particularly towards ourselves. So I think when you've spent however many years being critical, rewiring your brain takes time. And I like he says in the video, you know, it takes practise. It's not going to happen overnight. So yeah I think there are wider issues. 
And I think as well as being critical of yourself, you might think 'Oh God they're going to judge me, they're going to think this'." (Participant 9)

\subsection{Experiences and Relationships}

Participants talked about previous experiences and relationships having an impact on how they experienced the training. In particular, some talked about how self-compassion was not something that had been encouraged within their families and that more self-critical way of relating to themselves had been internalised from a young age. A few participants reflected that they felt that their generation were more open to the idea of self-compassion, but that some members of their family in older generations were more likely to see self-compassion as self-indulgent. This highlights social and historical differences in perspectives, which was also touched upon by a couple of participants who spoke of rigid gender constructs and roles contributing to men feeling less able to show vulnerability and compassion. The impact of relationships was sometimes felt to be positive, too: a few participants talked about previous therapy and other relationships helping them to already moved towards a more self-compassionate way of relating.

"If that's not something that you're that familiar with from your close family you're sort of a bit defensive against that, and then doing that yourself can actually almost bring up like a sense of loss and grief that 'This is new, this is novel, I haven't experienced this before. How lovely would it have been to have that?'”' (Participant 4)

\subsection{Prior Understanding}

Another part of participants' contexts and resources that influenced their experience was their level of prior understanding of self-compassion. Many participants reflected that their familiarity with self-compassion helped them to already see it in a favourable light and to readily understand the ideas being introduced in the training. The fact that some participants' familiarity with it involved an expectation that it might be a difficult process helped them to be patient with themselves in trying to engage with it. For several of the participants, the training was part of a multi-pronged approach and it helped them to build on changes they had already been trying to make. Some participants wondered whether the training might have been harder to understand and practice without their prior understanding as a resource, and a couple of participants said that they felt that they would have liked more time to grasp certain ideas.

"For me, it's part of a broader picture of all the different things that are interlinked, that they're all important and it's part of that. So for me, it's part of learning to be more giving, less ego, all that kind of thing, like a softening up." (Participant 13)

\subsection{Timing}

One of the ways in which timing mattered for participants was in relation to their mood and its impact on their level of motivation for engaging in the training. For example, some participants felt that the training coming at a time of great need fostered commitment to getting something out of the training, while others felt that their difficulties were too all-consuming for them to be able to engage with it. Some participants reflected that feeling good made it harder for them to muster motivation as there did not seem to be such a burning need, while others felt that this was 
something that helped them feel able to engage. This sub-theme also related to the previous one, in that some participants felt that they would not necessarily have been able to complete the training on their own terms if they had come across it at an earlier point in their lives. There were also some more practical issues in relation to timing, in terms of the other demands in people's lives and how possible it was to fit in the training around these.

"I think it's almost like I think if I wasn't in the best place when doing the training, I wouldn't have been looking after myself as well. I wouldn't set up that routine. That would be my worry is that sometimes, you know what you need to do but when you're not feeling great, that's when it's tested. That's when it's harder to put into place." (Participant 9)

\section{Discussion}

This study showed that self-compassion training offered in an online self-help format can be experienced as helpful by the general population. Most participants interviewed described having a positive overall experience of the training and they gave rich descriptions of ways in which the training made a difference in various areas of their lives, particularly in terms of their 'way of being' with themselves and others. Given the number of research studies which have demonstrated links between self-compassion and wellbeing [43-45], it is encouraging that self-help formats could offer a route to increasing the accessibility and scalability of CFT and CMT-based approaches for the general population.

One of the most striking findings was that many participants experienced a noticeable change in their way of relating to others as well as themselves. These findings tie in with Gilbert's view of compassion as a social mentality [13] and research on the inter-connectedness of different 'flows' of compassion: for others, from others and for oneself [46]. Irons and Heriot-Marland's study found that an eight-week CMT group led to stronger correlations between these three flows of compassion [16] and the frequency with which other qualitative CFT research has also found that participants describe a positive impact on their relationships $[11,12,15]$ suggests that this a significant strength of CFT-based approaches.

Participants also described developing more of an Observing self, bringing Compassionate intention to their everyday lives and engaging in more Compassionate self-talk. These sub-themes fit closely with CFT literature on the two mindsets or 'psychologies' of compassion, which Gilbert defines as "engagement with, and the alleviation/prevention of, suffering" (p. 26) [13]. These findings add weight to previous qualitative CFT research which has similarly found that participants described improvements in their capacity to step back from their difficulties and relate to themselves in kinder, non-blaming ways $[8,10,47]$.

Several of this study's themes pertain to facilitators and barriers to change which participants encountered when trying to develop a more self-compassionate way of being. These ranged from the accessibility of the training materials to wider-level factors such as the impingement of other demands in their lives, previous experiences which had influenced their ways of relating to themselves, and cultural beliefs around self-compassion. These themes highlight how challenging the process of developing self-compassion can be, and suggest that there needs to careful thought given to how appropriate a self-help approach is for individuals. It seems likely that the lack of personalised support would pose a significant barrier for some people, so it would be helpful to design online self-compassion training with variable degrees of guided support. Future research 
could compare the experience and effectiveness of unguided and guided CMT. While online guided self-help is now well-established in many NHS services, healthcare providers and other organisations offering such an approach would inevitably have to weigh up the benefits of guided support with the costs of the extra resources required. It could be valuable to have both guided and unguided versions of the self-compassion training could be offered, with the selection guided by people's preferences and circumstances.

The sub-theme Worries about self-compassion demonstrates that self-compassion can elicit ambivalent reactions for people attempting to engage with it. These included worries that it would have a negative impact on their relationships and work, a feeling that they did not deserve selfcompassion, and sadness when they realised how different self-compassion was from what they usually experienced from themselves and others. This latter experience has echoes of what psychoanalyst Patrick Casement calls the pain of contrast, referring to how painful it can be for people to find something that has been previously experienced as missing, and to get a glimpse of how it could have been to have had available when it was most needed [48]. These findings add further support to fears of compassion being a well-established phenomenon that has now been repeatedly found in compassion-related research $[2,3,7]$. It seems crucial that psychotherapeutic approaches think about how to target these fears. Compassion-focused approaches could be hypothesised to serve the function of gradually exposing people to these fears, and some studies have found reductions in fears of compassion [49].

The global COVID-19 pandemic and UK lockdowns were mentioned by numerous participants as a broader context which influenced their wellbeing and ability to engage. People's social and material living conditions, and their differing levels of access to power and resources, do not always get sufficiently incorporated into psychotherapeutic approaches. In line with some community psychology approaches, Smail argued that there is a danger of services and practitioners being excessively individualistic and 'voluntaristic' (i.e. suggesting that people can will their way out of difficulties) [50]. He called for there to be greater emphasis on therapeutic work including careful exploration of people's access to power and resources (e.g. through 'power mapping') [51] and appreciation of wider-level factors that can help or hinder people's difficulties, such as access to community support, supportive relationships, secure housing and employment. It is important that changes at individual level are not disproportionately emphasised the expense of efforts being made to develop more compassionate ways of relating to each other at a societal level.

\subsection{Limitations}

Given that most participants invited to an interview did not respond, it is possible that the interview data only comes from those who were particularly committed to the training and/or had more positive experiences of the training. However, online-based interventions are known to often have high rates of attrition [52] and it is hard to determine the extent to which attrition rates are related to participants' experience of this specific training or other factors.

Another limitation related to the sample is its composition of mostly white British participants with high levels of education. Generalisability of findings is generally not aimed for in qualitative research as much as it is in quantitative research, as small samples preclude representativeness, and its focus is instead on rich detail of the phenomena being explored. Nonetheless, the lack of diversity in the sample indicates that some voices are inadvertently being privileged over others. 
More research is needed to explore how the training is experienced by participants from a broader range of cultural contexts. While we attempted to reach people from a broad range of backgrounds through our advertising of the study, we still relied on convenience sampling and this may not be appropriate for the purpose of improving representativeness in research.

This study also lacked any follow-up interviews, which could have shed light on the extent to which the training had a lasting impact for participants and what it was like for people to try to continue on their own terms.

\section{Conclusions}

This has been the first qualitative study exploring participants' experience of engaging in an online self-help approach based on CMT, drawn from the CFT model. The findings show that the self-compassion training was experienced as helpful by participants, many of whom described developing a compassionate mentality that had a positive impact on their ways of relating to themselves and others. This provides support to the growing body of literature demonstrating the effectiveness of CMT and CFT-based approaches, and adds weight to Gilbert's suggestion that compassion should be seen as a social mentality [13]. The potential for a social ripple effect seems to be a particularly striking strength of CFT-based approaches. The fact that participants experienced similar benefits to those described in studies of CFT offered in individual and group formats indicates that online approaches could provide a way of increasing the accessibility and scalability of CMT and CFT. More broadly, these findings also have encouraging implications for other online approaches.

While the training was generally experienced positively, participants described encountering a diverse range of difficulties with the process. Some of these difficulties consolidated existing CFT literature (e.g. on fears of compassion), while others provided fresh perspectives that have not been explored in previous studies (e.g. in relation to the self-help format). The diverse range of facilitators and barriers to change highlight how differently approaches can be experienced by people, and suggest a need for a contextualised approach to be taken by healthcare providers when determining the potential suitability and helpfulness of approaches. Numerous participants described their social contexts influencing their experience of the training, which suggests that there is also a need for wider-level changes in order to better facilitate people developing more compassionate relationships with themselves and each other.

\section{Acknowledgments}

Thanks to Chloe Gibbons for conducting bracketing interviews with me, and Martha von Werthern for co-coding two interviews. Thanks to Michelle Williams for helping us to conceive the project.

\section{Author Contributions}

$\mathrm{JD}$ and $\mathrm{Cl}$ collaboratively recruited for our studies and set up the online self-compassion training on Qualtrics. JD and CN conducted their respective data collection and analysis processes separately: while JD carried out semi-structured interviews and analysed the interview data, CN collected quantitative data and analysed this. $\mathrm{Cl}$ developed the training materials used, and together with JK, 
he supervised all stages of the research project. $\mathrm{Cl}$ was not involved in the collection nor analysis of the data.

\section{Funding}

This study was funded by UCL Division of Psychology and Language Sciences. The research was undertaken as a requirement of JD and CN's Doctorates in Clinical Psychology. Their places on this programme were funded by Health Education England.

\section{Competing Interests}

The intervention outlined in this paper has been developed to be accessible to the general public via Balanced Minds. Due to this, $\mathrm{Cl}$ was not involved in the collection nor analysis of the data.

\section{References}

1. Gilbert $P$, Procter $S$. Compassionate mind training for people with high shame and self-criticism: Overview and pilot study of a group therapy approach. Clin Psychol Psychother. 2006; 13: 353379.

2. Gilbert P. Compassion: Conceptualisations, research and use in psychotherapy. London: Routledge; 2005.

3. Pauley G, McPherson S. The experience and meaning of compassion and self-compassion for individuals with depression or anxiety. Psychol Psychother. 2010; 83: 129-143.

4. Gilbert P, McEwan K, Matos M, Rivis A. Fears of compassion: Development of three self-report measures. Psychol Psychother. 2011; 84: 239-255.

5. Kirby J, Day J, Sagar V. The 'flow' of compassion: A meta-analysis of the fears of compassion scales and psychological functioning. Clin Psychol Rev. 2019; 70: 26-39.

6. Clapton NE, Williams J, Griffith GM, Jones RS. 'Finding the person you really are ... on the inside': Compassion focused therapy for adults with intellectual disabilities. J Intellect Disabil. 2017; 22: 135-153.

7. Lawrence VA, Lee D. An exploration of people's experiences of compassion-focused therapy for trauma, using interpretative phenomenological analysis. Clin Psychol Psychother. 2013; 21: 495-507.

8. Mullen G, Dowling C, Doyle J, O'Reilly G. Experiences of compassion focused therapy in eating disorder recovery: A qualitative model. Couns Psychother Res. 2020; 20: 248-262.

9. Ashworth F, Clarke A, Jones L, Jennings C, Longworth C. An exploration of compassion focused therapy following acquired brain injury. Psychol Psychother. 2015; 88: 143-162.

10. Bratt AS, Svensson I, Rusner M. Finding confidence and inner trust as a parent: Experiences of group-based compassion-focused therapy for the parents of adolescents with mental health problems. Int J Qual Stud Health Well-being. 2019; 14: 1684166.

11. Bell T, Dixon A, Kolts R. Developing a compassionate internal supervisor: Compassion-focused therapy for trainee therapists. Clin Psychol Psychother. 2017; 24: 632-648.

12. Gale C, Schröder T, Gilbert P. 'Do you practice what you preach?' A qualitative exploration of therapists' personal practice of Compassion Focused Therapy. Clin Psychol Psychother. 2017; 24: 171-185. 
13. Gilbert P. The origins and nature of compassion focused therapy. Br J Clin Psychol. 2014; 53: 641.

14. Neff K. Self-compassion: An alternative conceptualization of a healthy attitude toward oneself. Self Identity. 2003; 2: 85-101.

15. Carter A, Gilbert P, Kirby JN. Compassion-focused therapy for body weight shame: A mixed methods pilot trial. Clin Psychol Psychother. 2021; 28: 93-108.

16. Irons C, Heriot-Maitland C. Compassionate mind training: An 8-week group for the general public. Psychol Psychother. 2020; 94: 443-463.

17. Chamberlain $D$, Heaps $D$, Robert I. Bibliotherapy and information prescriptions: A summary of the published evidence-base and recommendations from past and ongoing books on prescription projects. J Psychiatr Ment Health Nurs. 2008; 15: 24-36.

18. Cuijpers $P$, Schuurmans J. Self-help interventions for anxiety disorders: An overview. Curr Psychiatry Rep. 2007; 9: 284-290.

19. Cavanagh K, Strauss C, Forder L, Jones F. Can mindfulness and acceptance be learnt by selfhelp?: A systematic review and meta-analysis of mindfulness and acceptance-based self-help interventions. Clin Psychol Rev. 2014; 34: 118-129.

20. Gellatly J, Bower P, Hennessy S, Richards D, Gilbody S, Lovell K. What makes self-help interventions effective in the management of depressive symptoms? Meta-analysis and metaregression. Psychol Med. 2007; 37: 1217-1228.

21. Halamová J, Kanovský M, Pačutová A, Kupeli N. Randomised controlled trial of an online version of compassion mind training in a nonclinical sample. Eur J Psychol. 2020; 16: 262-279.

22. Kelly A, Carter J. Self-compassion training for binge-eating disorder: A pilot randomized control trial. Psychol Psychother. 2015; 88: 285-303.

23. Kelly A, Waring S. A feasibility study of a two-week self-compassionate letter-writing intervention for non-treatment seeking individuals with typical and atypical anorexia nervosa. Int J Eat Disord. 2018; 51: 1005-1009.

24. Matos M, Duarte C, Duarte J, Pinto-Gouveia J, Petrocchi N, Basran J, et al. Psychological and physiological effects of compassionate mind training: A pilot randomized controlled study. Mindfulness. 2017; 8: 1699-1712.

25. Shapira LB, Mongrain M. The benefits of self-compassion and optimism exercises for individuals vulnerable to depression. J Posit Psychol. 2010; 5: 377-389.

26. Sommers-Spijkerman MP, Trompetter HR, Schreurs KM, Bohlmeijer ET. Compassion-focused therapy as guided self-help for enhancing public mental health: A randomized controlled trial. J Consult Clin Psychol. 2018; 86: 101-115.

27. Braun V, Clarke V. Successful qualitative research: A practical guide for beginners. California: Sage; 2013.

28. Gilbert P. The compassionate mind: A new approach to life's challenges. London: Constable \& Robinson; 2009.

29. Irons $C$, Beaumont $E$. The compassionate mind workbook: A step-by-step guide to developing your compassionate self. London: Robinson; 2017.

30. Kirby JN, Tellegen CL, Steindl SR. A meta-analysis of compassion-based interventions: Current state of knowledge and future directions. Behav Ther. 2017; 48: 778-792.

31. Kim JJ, Parker SL, Doty JR, Cunnington R, Gilbert P, Kirby JN. Neurophysiological and behavioural markers of compassion. Sci Rep. 2020; 10: 1-9. 
32. Gilbert P. Compassion focused therapy: The CBT distinctive features series. London: Routledge; 2010.

33. Roulston K. Considering quality in qualitative interviewing. Qual Res. 2010; 10: 199-228.

34. Braun V, Clarke V. Using thematic analysis in psychology. Qual Res Psychol. 2006; 3: 77-101.

35. Nowell LS, Norris JM, White DE, Moules NJ. Thematic analysis: Striving to meet the trustworthiness criteria. Int J Qual Methods. 2017; 16: 1609406917733847.

36. Saldaña J. The coding manual for qualitative researchers. California: Sage; 2009.

37. Braun V, Clarke V. Reflecting on reflexive thematic analysis. Qual Res Sport Exerc Health. 2019; 11: 589-597.

38. Fassinger R, Morrow SL. Toward best practices in quantitative, qualitative, and mixed-method research: A social justice perspective. J Soc Action Couns Psychol. 2013; 5: 69-83.

39. Terry G, Hayfield N, Clarke V, Braun V. Thematic analysis. In: The sage handbook of qualitative research in psychology. 2nd ed. California: Sage; 2017. pp. 17-37.

40. Bhaskar R. Reclaiming reality: A critical introduction to contemporary philosophy. London: Verso; 1989.

41. Danermark B, Ekstrom M, Jakobsen L, Karlsson J. Explaining society: Critical realism in the social sciences. London: Routledge; 2002.

42. Willig C. Introducing qualitative research in psychology: Adventures in theory and method. Maidenhead: Open University Press; 2008.

43. Craig C, Hiskey S, Spector A. Compassion focused therapy: A systematic review of its effectiveness and acceptability in clinical populations. Expert Rev Neurother. 2020; 20: 385-400.

44. Neff KD, Kirkpatrick KL, Rude SS. Self-compassion and adaptive psychological functioning. J Res Pers. 2007; 41: 139-154.

45. Van Dam NT, Sheppard SC, Forsyth JP, Earleywine M. Self-compassion is a better predictor than mindfulness of symptom severity and quality of life in mixed anxiety and depression. J Anxiety Disord. 2011; 25: 123-130.

46. Gilbert P, Catarino F, Duarte C, Matos M, Kolts R, Stubbs J, et al. The development of compassionate engagement and action scales for self and others. J compassionate health care. 2017; 4: 1-24.

47. Gooding H, Stedmon J, Crix D. 'All these things don't take the pain away but they do help you to accept it': Making the case for compassion-focused therapy in the management of persistent pain. Br J Pain. 2020; 14: 31-41.

48. Casement P. Learning from the Patient. New York: Guilford Publications; 1992.

49. Jazaieri H, Jinpa GT, McGonigal K, Rosenberg EL, Finkelstein J, Simon-Thomas E, et al. Enhancing compassion: A randomized controlled trial of a compassion cultivation training program. J Happiness Stud. 2013; 14: 1113-1126.

50. Smail D. The origins of unhappiness: A new understanding of personal distress. London: Routledge; 2015.

51. Hagan T, Smail D. Power-mapping-I. Background and basic methodology. J Community Appl Soc Psychol. 1997; 7: 257-267.

52. Eysenbach G. The law of attrition. J Medical Internet Res. 2005; 7: e402. 
OBM Integrative and Complementary Medicine 2021; 6(4), doi:10.21926/obm.icm.2104050

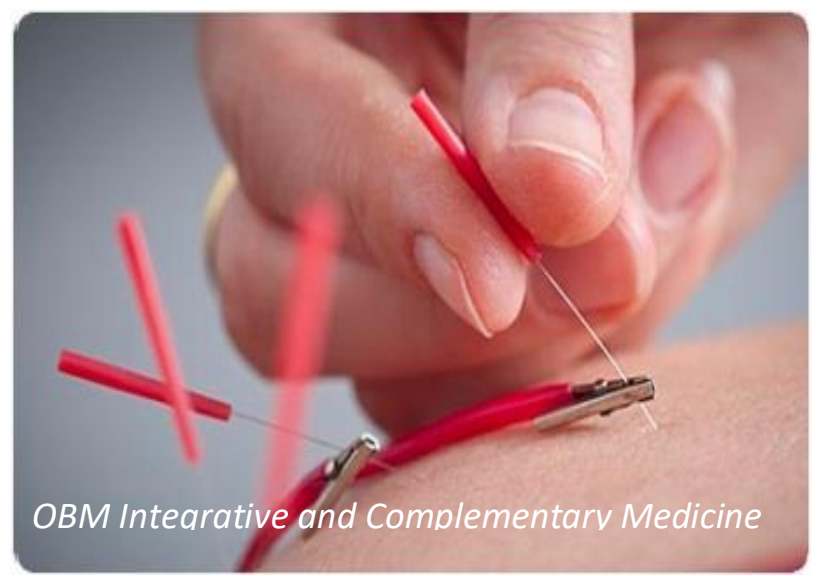

Enjoy OBM Integrative and Complementary Medicine by:

1. Submitting a manuscript

2. Joining in volunteer reviewer bank

3. Joining Editorial Board

4. Guest editing a special issue

For more details, please visit:

http://www.lidsen.com/journals/icm 